\title{
Determination of the Arbitrary Constants which appear in the Asymptotic Expansions for the Functions of the Elliptic Cylinder.
}

By William Marshall.

\section{(Read and Received 9th December 1921.)}

In an article, ${ }^{*}$ published some time since, the author of the present paper deduced an asymptotic expression for the functions of the elliptic cylinder, which expression took the following form:

$$
U=\frac{C}{e^{\frac{u}{2}}}\left[P \cos \left(a-\frac{k e^{u}}{2}\right)+Q \sin \left(a-\frac{k e^{u}}{2}\right)\right] .
$$

Here $P$ and $Q$ are certain asymptotic series, and $C$ and $a$ arbitrary constants. General expressions for these constants were not determined in the aforementioned article on account of the difficulties there set forth, though it was pointed out that their numerical calculation for any particular problem was simply a matter of arithmetic computation. It is the object of the present paper to deduce general expressions for these constants $C$ and $a$ in terms of the two parameters which appear in the defining equation for $U$.

The starting point in this investigation is an important and far-reaching result due to Whittaker, that the periodic solutions of the differential equation,

$$
\frac{d^{2} y}{d z^{2}}+\left(\alpha+k^{2} \cos ^{2} z\right) y=0
$$

satisfy the homogeneous integral equation,

$$
y(z)+\lambda \int_{0}^{2 \pi} e^{k \cos \theta \cos z} y(\theta) d \theta=0
$$

* Am. Jour. of Math. Vol. XXXI., (1909), p. 311.

†E. T. Whittaker. Ioternational Congress of Mathematicians, Cam. bridge, 1912. 
It is by means of this fact that we are enabled to express the functions of the elliptic cylinder in the form of definite integrals, and these lead easily to the desired end.

If the equation defining the functions of the elliptic cylinder be taken in the form,

$$
\frac{d^{2} U}{d u^{2}}+\left(k^{2} \cosh ^{2} u+\alpha\right) U=0,
$$

then the relations

$$
e^{n}=z, \frac{k z}{2}=x, U_{1}=U z^{t}
$$

transform (4) into

$$
\frac{d^{2} U_{1}}{d x^{2}}+\left(1+\frac{p}{x^{2}}+\frac{q}{x^{4}}\right) U_{1}=0
$$

where

$$
p=\frac{k^{2}}{2}+\alpha+\frac{1}{4}, q=\frac{k^{4}}{16} .
$$

An asymptotic solution for (6) is for large values of $x$,

$$
U_{1}=C[P \cos (a-x)+Q \sin (a-x)],
$$

where $P$ and $Q$ are given by

$$
\begin{aligned}
& P=1-\frac{f_{2}(p, q)}{x^{2}}+\frac{f_{4}(p, q)}{x^{4}}-\frac{f_{6}(p, q)}{x^{6}}+\ldots \\
& Q=-\frac{f_{1}(p, q)}{x}+\frac{f_{3}(p, q)}{x^{3}}-\frac{f_{6}(p, q)}{x^{5}}+\ldots
\end{aligned}
$$

and where $C$ and $a$ are to be so determined, that (8) is, for large values of $x$, an approximation to one of the fundamental integra]s of (6).

If now use is made of a theorem similar to Whittaker's Theorem mentioned above, it is found that a solution of (6) can be expressed in the form of the definite integral

$$
U_{1}=\left(\frac{2 x}{k}\right)^{\frac{3}{2}} \int_{0}^{2 \pi} \cos \left[\left(x+\frac{k^{2}}{4 x}\right) \cos \theta\right] S(\theta) d \theta,
$$

*Am. Joumal of Math. Vol. XXXI. (1909) p. 311, et seq. 
procided that $S(\theta)$ is a periodic solution, of period $2 \pi$ of the equation

$$
\frac{d^{2} S}{d \theta^{2}}-\left(k^{2} \cos ^{2} \theta+\alpha\right) S=0 .
$$

The periodic solutions of an equation differing but slightly from equation (11) form the subject of an article by Dannacher.* Following this method, if we change the constants in equation (11) by putting,

$$
k^{2}=16 \beta^{2}, \alpha=4 m-8 \beta^{2},
$$

equation (11) goes over into

$$
\frac{d^{2} S}{d \theta^{2}}-\left(8 \beta^{2} \cos 2 \theta+4 m\right) S=0 .
$$

A periodic solution of this equation is

$$
S(\theta)=\frac{1}{2} a_{0}+a_{1} \cos 2 \theta+a_{2} \cos 4 \theta+\ldots
$$

in which $a_{0}$ is arbitrary, and in which the following recurrence formulas hold for the other a's.

$$
\begin{aligned}
& a_{1}=-\frac{m a_{0}}{2 \beta^{2}} \\
& a_{2}=-a_{0}-\frac{a_{1}}{4 \beta^{2}}\left(4 m+2^{2}\right) \\
& a_{3}=-a_{1}-\frac{a_{2}}{4 \beta^{2}}\left(4 m+4^{2}\right) \\
& \ldots \ldots \ldots \ldots \ldots \ldots \ldots \ldots \ldots \ldots \ldots \ldots \ldots \ldots \ldots \ldots \ldots \ldots \ldots \ldots \ldots \\
& a_{n}=-a_{n-2}-\frac{a_{n-1}}{4 \beta^{2}}\left[4 m+(2 n-2)^{2}\right],
\end{aligned}
$$

and in which also,

$$
\begin{aligned}
m & =\frac{2 \alpha+k^{2}}{8} \\
\beta^{2} & =\frac{k^{2}}{16} .
\end{aligned}
$$

It may be remarked, though this is foreign to our present purpose, that the series (14) is not convergent for all values of $m$ and $\beta$,

* S. Dannacher. Diss. Züirich, 1906. 
but only for certain special values, which appear as the roots of a certain "limiting function" containing the constants $m$ and $\beta$." We have then that a solution of

$$
\frac{d^{2} U_{1}}{d x^{2}}+\left[1+\left(\frac{k^{2}}{2}+\alpha+\frac{1}{4}\right) \cdot \frac{1}{x^{2}}+\frac{k^{4}}{16 x^{4}}\right] U_{1}=0
$$

may be expressed in the form of the definite integral

$$
U_{1}=\left(\frac{2 x}{k}\right)^{\frac{1}{2}} \int_{0}^{2 \pi} \cos \left[\left(x+\frac{k^{2}}{4 x}\right) \cos \theta\right] S(\theta) d \theta,
$$

where $S(\theta)$ is given by (14) together with the relations (15) and (16). The problem is now to determine the approximate value of the integral in (10) as $x$ increases. To do this, in (10) put $\cos \theta=1-s$, then on splitting up the interval from 0 to $2 \pi$ into the intervals 0 to $\pi$, and $\pi$ to $2 \pi,(10)$ goes over into

$$
U_{1}=2\left(\frac{2 x}{k}\right)^{\frac{1}{2}} \int_{0}^{2} \cos \left[\left(x+\frac{k^{2}}{4 x}\right)(1-s)\right] T(s) \frac{d s}{\sqrt{2 s-8^{2}}}
$$

where $T(s)$ is the expression obtained when in (14) $\cos \theta$ is replaced by $1-s$.

Now since the cosines of even multiples of an angle can be expressed as polynomials in even powers of the cosines of the angle, and since moreover the sum of the coefficients in such a polynomial equals unity, $T(s)$ must have the form

$$
T(s)=\frac{1}{2} a_{0}+a_{1} p_{1}+a_{2} p_{2}+\ldots
$$

where $p_{1}, p_{2}, p_{3} \ldots$ are polynomials in $s$, each of the form $1+$ powers of $s$.

In equation (17) put now $s x=t$; then (17) becomes

$$
\begin{aligned}
U_{1}=\left(\frac{2}{k}\right)^{\frac{1}{2}} 2 \int_{0}^{2 x} \cos \left[\left(x+\frac{k^{2}}{4 x}\right)\left(1-\frac{t}{x}\right)\right] \\
{\left[\frac{1}{2} a_{0}+a_{1} q_{1}+a_{2} q_{2}+\ldots\right] \frac{d t}{\sqrt{2 t-\frac{t^{2}}{x}}} }
\end{aligned}
$$

where $q_{1}, q_{2}, q_{3} \ldots$ are polynomials of the form $1+$ powers of $\frac{t}{x}$.

- See Dannacher, l. $c_{.}$, for a study of this function in the case of the related equation. 
The integral (19) may now be written

$$
\begin{aligned}
& U_{1}=\left(\frac{2}{k}\right)^{t} 2 \int_{0}^{2 x} \cos \left(x+\frac{k^{2}}{4 x}\right) \cos \left(t+\frac{t k^{2}}{4 x^{2}}\right)\left(\frac{1}{2} a_{0}+a_{1} q_{1}+\ldots\right) \frac{d t}{\sqrt{2 t-\frac{t^{2}}{x}}} \\
&+\left(\frac{2}{k}\right)^{\frac{1}{2}} 2 \int_{0}^{2 x} \sin \left(x+\frac{k^{2}}{4 x}\right) \sin \left(t+\frac{t k^{2}}{4 x^{2}}\right) \\
&\left(\frac{1}{2} a_{0}+a_{1} q_{1}+\ldots\right) \frac{d t}{\sqrt{2 t-\frac{t^{2}}{x}}}
\end{aligned}
$$

and for large values of $x$ this becomes approximately,

$$
\begin{aligned}
U_{1} & =\left(\frac{2}{k}\right)^{\frac{1}{2}} 2 \int_{0}^{\infty} \cos x \cos t\left(\frac{1}{2} a_{0}+a_{1}+a_{2}+\ldots\right) \frac{d t}{\sqrt{\frac{2 t}{2}}} \\
& +\left(\frac{2}{k}\right)^{\frac{1}{2}} 2 \int_{0}^{\infty} \sin x \sin t\left(\frac{1}{2} a_{0}+a_{1}+a_{2}+\ldots\right) \frac{d t}{\sqrt{2 t}} .
\end{aligned}
$$

This may be written finally

$$
\begin{aligned}
U_{1}=\frac{2}{k^{2}}\left(\frac{1}{2} a_{0}+a_{1}+a_{2}+\ldots\right) & \\
& \left(\cos x \int_{0}^{\infty} \frac{\cos t d t}{\sqrt{t}}+\sin x \int_{0}^{\infty} \frac{\sin t d t}{\sqrt{t}}\right) .
\end{aligned}
$$

The integrals in (29) are well-known, each being equal to $\sqrt{\frac{\pi}{2}}$.

There results then

$$
U_{1}=\frac{2}{k^{\downarrow}}\left(\frac{1}{2} a_{0}+a_{1}+a_{2}+\ldots\right)\left(\sqrt{\frac{\pi}{2}} \cos x+\sqrt{\frac{\pi}{2}} \sin x\right),
$$

which gives as the final approximate value of $U_{1}$, for large $x$,

$$
U_{1}=2 \sqrt{\frac{\pi}{k}}\left(\frac{1}{2} a_{0}+a_{1}+a_{2}+\ldots\right) \cos \left(\frac{\pi}{4}-x\right) .
$$

Returning to the expression for $U_{1}$ as given by

$$
U_{1}=C[P \cos (a-x)+Q \sin (a-x)]
$$


where

$$
\begin{aligned}
& P=1 \frac{f_{2}(p, q)}{x}+\frac{f_{4}(p, q)}{x^{4}}-\ldots \\
& Q=-\frac{f_{1}(p, q)}{x}+\frac{f_{3}(p, q)}{x^{3}}-\ldots
\end{aligned}
$$

it is evident that (6) becomes for large values of $x$, approximately,

$$
U_{1}=C^{\prime} \cos (a-x) \text {. }
$$

Comparing this with (24) the desired results are obtained, namely,

$$
\begin{aligned}
& C=2 \sqrt{\frac{\pi}{k}}\left(\frac{a_{0}}{2}+a_{1}+a_{2}+\ldots\right), \\
& a=\frac{\pi}{4} .
\end{aligned}
$$

It may be noted that the expression for $U_{1}$ as given by (24) can also be deduced from a result of Heine's. *

The equation studied by Heine is

$$
\frac{d^{2} E}{d \phi^{2}}+\left(\lambda^{2} \cos ^{2} \phi-L\right) E=0 .
$$

If in this we put $i \phi=u, \lambda^{2}=-k^{2}$ and replace $L$ by $\alpha$ and $E$ by $U$, equation (27) goes over into

$$
\frac{d^{2} U}{d u^{2}}+\left(k^{2} \cosh ^{2} u+\alpha\right) U=0
$$

which is exactly equation (4).

Now, a solution of (27), as Heine shows, can be expressed in terms of Bessel's functions of even order, thus,

$$
E(\phi)=\frac{1}{2} a_{0} J_{0}(i \lambda \cos \phi)-a_{1} J_{2}(i \lambda \cos \phi)+a_{2} J_{4}(i \lambda \cos \phi)-\ldots
$$

If in this there be substituted $i \phi=u, \lambda=k i$, and if $E$ is replaced by $U$, th re appears a solution of (28) or (4) in the form,

$$
U=\frac{1}{2} a_{\theta} J_{0}(-k \cosh u)-a_{1} J_{2}(-k \cosh u)+\ldots
$$

In this make the transformations (5), then a solution of

$$
\frac{d^{2} U_{1}}{d x^{2}}+\left(1+\frac{p}{x^{2}}+\frac{q}{x_{1}}\right) U=0
$$

* E. Heine. Handbuch der Kugtlfunktionen. 2te Auf. Band I. S. 414. 
has the form

$$
U_{1}=\left(\frac{2 x}{k}\right)^{\frac{1}{2}}\left[\frac{1}{2} a_{0} J_{0}\left(x+\frac{k^{2}}{4 x}\right)-a_{1} J_{2}\left(x+\frac{k^{2}}{4 x}\right)+\ldots\right]
$$

For large values of $x$ this becomes approximately, using the asymptotic expressions for $J_{0}, J_{2}, \ldots *$

$$
U_{1}=\left(\frac{2 x}{k}\right)^{\frac{1}{2}}\left[\frac{1}{2} a_{0} \sqrt{\frac{2}{\pi x}} \cos \left(\frac{\pi}{4}-x\right)-a_{1} \sqrt{\frac{2}{\pi x}} \cos \left(\frac{5 \pi}{4}-x\right)+\ldots\right]
$$

which reduces to

$$
U_{1}=\frac{2}{\sqrt{\pi k}} \cos \left(\frac{\pi}{4}-x\right)\left(\frac{1}{2} a_{0}+a_{1}+a_{2}+a_{3}+\ldots\right)
$$

and this differs only by a constant multiplier from the approximate value given by (24).

* Nielson. Handbuch $d$ Theorie d. Cylinderfunktionen, S. 156. 